\title{
CONTRIBUTION TO UNRESOLVED INFRARED FLUCTUATIONS FROM DWARF GALAXIES AT REDSHIFTS OF 2-3
}

\author{
Ranga-Ram Chary, ${ }^{1}$ Asantha Cooray, ${ }^{2}$ and Ian Sullivan ${ }^{1}$ \\ Received 2007 November 13; accepted 2008 March 25
}

\begin{abstract}
In order to understand the origin of clustered anisotropies detected in Spitzer images between 3.6 and $8 \mu \mathrm{m}$, we stack the Spitzer IRAC/Great Observatories Origins Deep Survey (GOODS) images at pixel locations corresponding to faint, $z_{\mathrm{AB}} \sim 27 \mathrm{mag}$, optical sources with no obvious IR counterparts. We obtain a strong detection of the sources with a stacked median flux at $3.6 \mu \mathrm{m}$ of $130 \pm 5 \mathrm{nJy}$ above the background. The wealth of multiwavelength imaging data in GOODS enables a similar stacking analysis to be undertaken at various wavelengths between the ultraviolet and near-infrared bands. We obtain strong stacked detections of these optically faint sources over the entire wavelength range, which places constraints on the average properties of these sources. We find that the flux spectrum of the median, stacked source is consistent with a $L \lesssim 0.03 L_{*}$, Uv galaxy with a $90 \%$ confidence interval for the redshift of 1.9-2.7. These sources produce a $3.6 \mu \mathrm{m}$ absolute background intensity between 0.1 and $0.35 \mathrm{nW} \mathrm{m} \mathrm{sr}^{-1}$, and the clustered IR light could account for $\sim 30 \%-50 \%$ of fluctuation power in the IR background at $4^{\prime}$ angular scales. Although the exact redshift distribution of these sources is unknown, these galaxies appear to contain $5 \%-20 \%$ of the comoving stellar mass density at $z \sim 2.5$.
\end{abstract}

Subject headings: diffuse radiation — infrared: galaxies — large-scale structure of universe

Online material: color figures

\section{INTRODUCTION}

The intensity of the cosmic near-infrared background (IRB) is a measure of the total light emitted by stars and galaxies in the universe which is not thermally reprocessed by dust. The absolute background has been estimated with the Diffuse Infrared Background Experiment (DIRBE; Hauser \& Dwek 2001) and the Infrared Telescope in Space (IRTS; Matsumoto et al. 2005), which results in values that are about a factor of 2 higher than the intensity obtained by integrating the light from individually detected galaxies. This is most likely attributable to the large uncertainties associated with the removal of foreground zodiacal light from the DIRBE observations (Dwek et al. 2005). This hypothesis has been strengthened by the $\mathrm{TeV}$ spectrum of gammaray blazars, which indicate that the total IRB intensity is smaller than the DIRBE IRTS estimates (Aharonian et al. 2006) and that $\sim 90 \%$ of the IRB light probably arises in known galaxy populations. Yet, attempts have been made to explain the difference between the measured and resolved IRB intensity with sources at the epoch of reionization (e.g., Santos et al. 2002; Salvaterra \& Ferrara 2003; Cooray \& Yoshida 2004; Fernandez \& Komatsu 2006). If first-light galaxies are to explain the missing intensity completely, then an extreme scenario is needed with the conversion of at least $5 \%$ of all baryons to stars (Madau \& Silk 2005).

Instead of the absolute intensity, recent works have concentrated on spatial fluctuations of the IRB (Cooray et al. 2004; Kashlinsky et al. 2004). While an interpretation of unresolved fluctuations is subject to extremely uncertain astrophysical modeling of underlying faint populations, fluctuations in deep Spitzer images have been fully attributed to first-light galaxies containing Population III stars during reionization (Kashlinsky et al. 2005). An alternative study shows that a reasonable fraction ( $>50 \%$ ) of unresolved fluc-

\footnotetext{
${ }^{1}$ Division of Physics, Mathematics, and Astronomy, California Institute of Technology, Pasadena, CA 91125; rchary@caltech.edu.

${ }^{2}$ Center for Cosmology, Department of Physics and Astronomy, University of California, Irvine, CA 92697; acooray@uci.edu.
}

tuations is arising from faint, unresolved sources at lower redshifts (Cooray et al. 2007). Thus, while two independent studies find a similar clustering amplitude for IRB fluctuations (Kashlinsky et al. 2004; Cooray et al. 2007), they differ in the interpretation related to the contribution from Population III stars due to differences in the background light ascribed to faint foreground galaxies unresolved by Spitzer.

The suggestion for a low-redshift origin in Cooray et al. (2007) comes from predictions using a halo model (Cooray \& Sheth 2002 ) for the $3.6 \mu \mathrm{m}$ population matched to their luminosity functions (Babbedge et al. 2006) and clustering power spectra (Sullivan et al. 2007). In Cooray et al. (2007) this low-redshift population was identified as the faint optical galaxies that are resolved in the Hubble Space Telescope Advanced Camera for Surveys (HST ACS; Giavalisco et al. 2004) images of the Great Observatories Origins Deep Survey (GOODS; Dickinson et al. 2003a) fields, but not detected in deep Spitzer Infrared Array Camera (IRAC) images of the same fields. With models, it was estimated that this faint galaxy population has a $3.6 \mu \mathrm{m}$ intensity between 0.1 and $0.8 \mathrm{nW} \mathrm{m}^{-2} \mathrm{sr}^{-1}$. The analysis in Cooray et al. (2007) did not exclude a high-redshift contribution to the IRB, with an upper limit on the $3.6 \mu \mathrm{m}$ absolute background intensity from $z>8$ sources of $0.6 \mathrm{nW} \mathrm{m}{ }^{-2} \mathrm{sr}^{-1}$. In comparison, if all unresolved IR fluctuations are generated by faint $z>6.5$ sources which are below the detection threshold of Spitzer, then the IRB intensity for such sources is $\gtrsim 1 \mathrm{nW} \mathrm{m}^{-2} \mathrm{sr}^{-1}$ at $3.6 \mu \mathrm{m}$ (Kashlinsky et al. 2007c).

In this paper, we further study the physical origin of fluctuations in the IRB. Since faint ACS optical sources are undetected in deep Spitzer IRAC images, we stack their pixel locations in IRAC to establish the average IR flux. We also utilize the wealth of multiwavelength imaging data in the GOODS fields to obtain a stacked broadband spectral energy distribution (SED) of these faint optical galaxies between UV and near-IR bands. Leaving the redshift as a free parameter, we fit the average optical to IR flux spectrum with galaxy population synthesis models and find that 


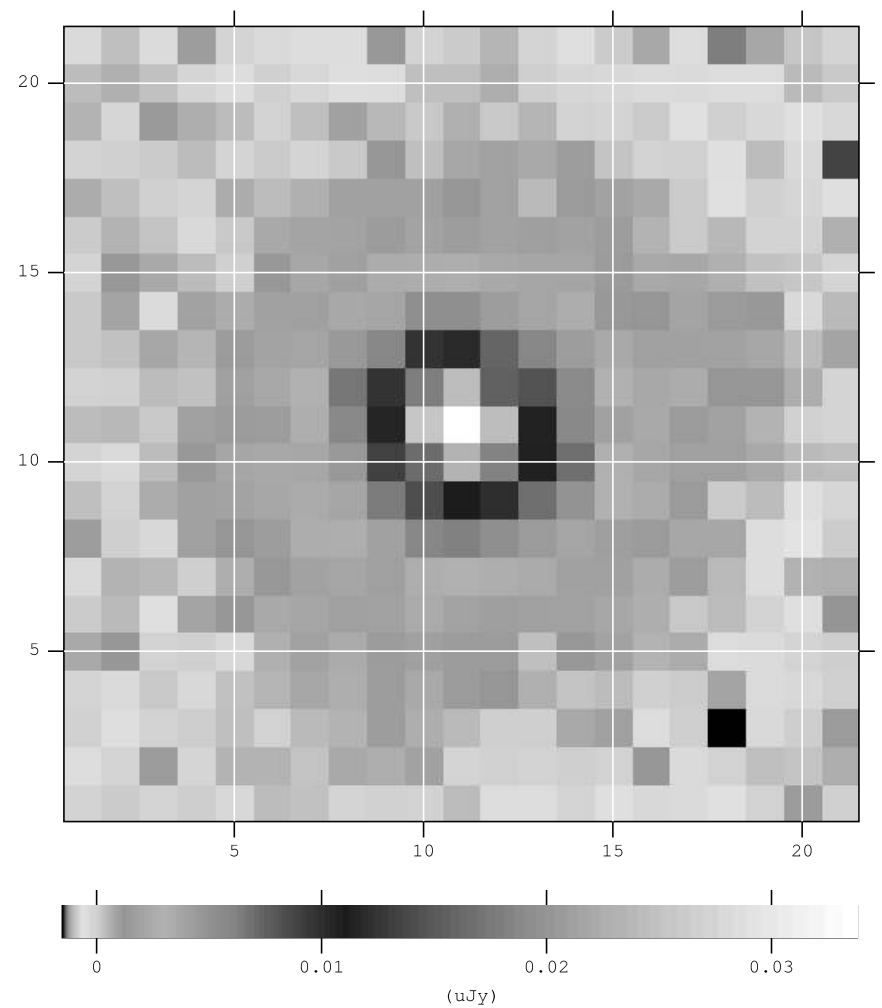

FIG. 1.- Average-stacked 3.6 $\mu \mathrm{m}$ image from GOODS-N with pixel locations of all 6160 ACS sources unmatched and unmasked in IRAC. The median stacked flux is $(130 \pm 5) \mathrm{nJy}$ (or $=26.1 \pm 0.1 \mathrm{mag}$ ) and is detected with a statistical signal-to-noise ratio higher than 25. [See the electronic edition of the Journal for a color version of this figure.]

the stacked flux spectrum is best fit by a $\sim 0.03 L_{*, \mathrm{UV}}$ galaxy at $1.9<z<2.7$, similar to a scaled-down population of Lyman break galaxies. We also measure the expected IRB fluctuation clustering spectrum from these sources.

We summarize the stacking analysis in the next section and discuss results on the average optical to IR flux spectrum in $\S 3$. We discuss clustering of these faint optical sources in $\S 4$. In $\S 5$ we discuss the implications of our measurements. The magnitudes quoted throughout this paper are AB magnitudes.

\section{STACKING ANALYSIS}

To establish the average IR intensity of faint optical sources that are unresolved in Spitzer IRAC images we stack the IRAC images at the spatial coordinates of faint, optical sources which are detected in the GOODS optical data. The stacking begins by first masking all $>3 \sigma$ detected IRAC sources with $m_{\mathrm{AB}}(3.6 \mu \mathrm{m})<$ $26.7 \mathrm{mag}$ in the GOODS IRAC mosaics. ${ }^{3}$ This limit is fainter than the $50 \%$ completeness limit of $24.7 \mathrm{mag}$. The mask has a radius of $13.5^{\prime \prime}$ for a source with 18 mag and scales linearly with magnitude down to $2.4^{\prime \prime}$ for sources fainter than 22 mag. We then identified sources in the GOODS ACS catalogs (Giavalisco et al. 2004) which are in unmasked regions of the IRAC mosaics and are therefore IRAC undetected. While there are close to 22,000 ACS sources which are unmatched with IRAC sources to within $\sim 0.5^{\prime \prime}$, only 6160 (GOODS-N) and 5441 (GOODS-S) sources are sufficiently separated from brighter IRAC sources so as to be in unmasked regions of the IRAC image. The IRAC images for each GOODS field were stacked at the pixel locations

\footnotetext{
3 A description of the GOODS mosaics can be found at http://ssc.spitzer .caltech.edu/legacy/.
}

TABLE 1

Results from Stacking Analysis

\begin{tabular}{|c|c|c|}
\hline Parameter & GOODS-N & GOODS-S \\
\hline$\Delta \Omega / \operatorname{arcmin}^{2}$. & 169 & 171 \\
\hline$N_{\mathrm{ACS}}$ & 6160 & 5441 \\
\hline U & $28.3 \pm 0.1$ & $28.4 \pm 0.1$ \\
\hline В & $28.0 \pm 0.1$ & $27.8 \pm 0.1$ \\
\hline$V \ldots \ldots \ldots \ldots \ldots \ldots \ldots$ & $27.5 \pm 0.1$ & $27.4 \pm 0.1$ \\
\hline$i$ & $27.2 \pm 0.1$ & $27.1 \pm 0.1$ \\
\hline$z$ & $27.1 \pm 0.1$ & $27.0 \pm 0.1$ \\
\hline$H K^{\prime} \ldots \ldots$ & $27.3 \pm 0.3$ & $\ldots$ \\
\hline J & $\ldots$ & $26.9 \pm 0.15$ \\
\hline Н & $\ldots$ & $26.7 \pm 0.15$ \\
\hline 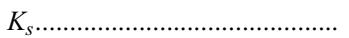 & $\ldots$ & $26.6 \pm 0.15$ \\
\hline $3.6 \mu \mathrm{m}$ & $26.1 \pm 0.1$ & $26.0 \pm 0.1$ \\
\hline $4.5 \mu \mathrm{m}$ & $26.2 \pm 0.1$ & $26.2 \pm 0.1$ \\
\hline $5.8 \mu \mathrm{m}$ & $26.2 \pm 0.1$ & $26.0 \pm 0.1$ \\
\hline $8.0 \mu \mathrm{m}$ & $26.3 \pm 0.15$ & $26.0 \pm 0.15$ \\
\hline
\end{tabular}

Notes.-The expression $N_{\mathrm{ACS}}$ is the total number of faint ACS sources over an effective area $\Delta \Omega$ that were included in the stack. Uncertainties in the stacked flux (tabulated in $\mathrm{AB}$ magnitudes) are mostly dominated by calibration systematics.

corresponding to these $\sim 6000$ ACS sources. The stacking was undertaken by making $12^{\prime \prime}$ image cutouts from the masked IRAC mosaic, centered on each ACS source position. The cutouts were then co-added using a $3 \sigma$ clipped median, resulting in a median stacked image of the sources, as shown in Figure 1. A count of the number of sources which contribute to the stacked flux is also maintained, resulting in an effective exposure map. Pixels which have been masked do not contribute either flux or exposure in the stacking process. Circular aperture photometry was performed on this stacked image, and the flux measured in a $3.6^{\prime \prime}$ radius beam. The sky background was estimated from the stacked image by measuring the sigma-clipped median of pixels within a surrounding sky annulus spanning $5^{\prime \prime}-6^{\prime \prime}$. The profile of the source had a full width at half-maximum of $1.6^{\prime \prime}$ and was consistent with an unresolved point source at the resolution of Spitzer. Aperture corrections were estimated by performing identical photometry on a calibration point source and correspond to a factor of 1.14. After background subtraction and aperture corrections to the photometry were performed, the resulting stacked $3.6 \mu \mathrm{m}$ flux density is $130 \pm 1.3 \mathrm{nJy}$.

In order to assess the reliability of the stacked flux, we stacked the IRAC images at an identical number of positions which are offset from the nominal ACS source positions by random amounts, up to $9^{\prime \prime}$ from the original source coordinates. This has the advantage of accounting for any possible contamination of the stacked flux due to the wings of nearby sources which may not be completely masked, while measuring the same local sky background. The random stacks were repeated 100 times and the flux of the stack measured. The standard deviation of these values results in a flux density of $5 \mathrm{nJy}$, which is a factor of 4 larger than the uncertainty simply due to the background noise term due to source confusion and the low-level flux from the wings of masked sources. Thus, the stacking results in a strong detection of these faint optical galaxies, and we adopt a stacked $3.6 \mu \mathrm{m}$ flux density of $130 \pm 5 \mathrm{nJy}$.

This procedure was repeated at each IRAC wavelength independently for each GOODS field to assess the reliability of the result. Table 1 summarizes stacking the results for all IRAC wavelengths between 3.6 and $8.0 \mu \mathrm{m}$. Through the stacking analysis, we obtain a strong detection of these faint optical galaxies and 
measure a flat flux spectrum $\left(F_{\nu} \propto \nu^{0.08 \pm 0.3}\right)$. This is consistent with the flat frequency spectrum of IR fluctuations between 3.6 and $8.0 \mu \mathrm{m}$ found by Kashlinsky et al. (2005).

In order to estimate the total contribution to the IRB intensity of these sources, we multiply the median stacked flux by the exposure map. Based on the surface density of about 6000 galaxies in each GOODS field, we establish the absolute $3.6 \mu \mathrm{m}$ IR intensity of these faint optical sources to be $0.12 \pm 0.01 \mathrm{nW} \mathrm{m}^{-2} \mathrm{sr}^{-1}$. We note that this estimate is larger than the value obtained by simply multiplying the quoted number of sources $(\sim 6000)$ with the median flux. This is because the quoted number of sources requires that a source be unmasked out to a radius of $3.6^{\prime \prime}$, while the exposure map takes into account the exact number of sources which contribute to the stacked flux in each pixel. Since some of the sources may be partially masked within the $3.6^{\prime \prime}$ radius in the exposure map, the quoted number of sources is smaller than the number of sources as estimated from the exposure map.

Furthermore, this estimate of $0.12 \mathrm{nW} \mathrm{m}^{-2} \mathrm{sr}^{-1}$ only accounts for sources that remain outside the IRAC mask and are not confused with bright IRAC sources. Assuming the average flux from the stack also applies to sources affected by the mask, we estimate the absolute IRB intensity to be as high as $0.35 \mathrm{nW} \mathrm{m}^{-2} \mathrm{sr}^{-1}$. This is well within the range of $0.1-0.8 \mathrm{nW} \mathrm{m}^{-2} \mathrm{sr}^{-1}$ estimated for the contribution of faint sources below the individual point-source detection limit in IRAC images through clustering models (Sullivan et al. 2007).

To further study the nature of these sources, beyond Spitzer IRAC wavelengths, we adopted a similar procedure and stacked the same population at other wavelengths at which the GOODS fields have imaging data. Specifically, for GOODS-N, we utilize the KPNO Uband (Capak et al. 2004), HST ACS BViz (Giavalisco et al. 2004), and near-IR $H K^{\prime}$ (Capak et al. 2004). For GOODS-S, we use the CTIO $U$ band and the ESO ISAAC near-IR data in the $J H K$ bands in addition to the HST and Spitzer data. For the HST data, we simply measure the median flux values of the sources, since they are individually detected at the ACS wavelengths. For the other wavelengths, we excised subimages for each source and estimated a sigma-clipped average of the subimages to obtain the stacked flux, as was done for the IRAC data. Aperture corrections, background estimates, and noise calculations were performed as described for the IRAC data.

\section{REDSHIFT AND STELLAR MASS DENSITY ESTIMATES}

In order to understand the physical properties of these faint galaxies, we fit the stacked flux in Table 1 with Bruzual \& Charlot (2003) population synthesis models. Redshift, mass, extinction, age, and $e$-folding time of star formation were left as free parameters. The redshift range was $0.1-6$ in steps of 0.1 , extinction range was $A_{V}=0-5$ in steps of 0.1 , age $t$, varied from $10^{5} \mathrm{yr}$ to the age of the universe at each redshift, while the $e$-folding time $\tau$ spanned the entire range from instantaneous to constant star formation. For GOODS-N, we find a best-fit redshift of 1.9, stellar mass of $1.3 \times 10^{8} M_{\odot}, A_{V}=1.4 \mathrm{mag}, t=10 \mathrm{Myr}$, and $\tau=200 \mathrm{Myr}$, indicating an ongoing reddened starburst (Fig. 2). For GOODS-S, which has far better near-IR data, we find a bestfit redshift of 2.1, stellar mass of $1.4 \times 10^{8} M_{\odot}, A_{V}=1.1 \mathrm{mag}$, $t=15 \mathrm{Myr}$, and $\tau=200 \mathrm{Myr}$. The $90 \%$ confidence interval for the redshifts as determined from the lowest $\chi^{2}$ fit at each redshift, spans the range $1.9<z<2.9$ for GOODS-S and $1.3<$ $z<2.7$ for GOODS-N. The $U$-band detection implies that the majority of the sources must be at $z<2.5$. The sharp increase in the flux in the IRAC channels compared to the near-IR bands implies that the $1.6 \mu \mathrm{m}$ bump must be in the IRAC passbands.

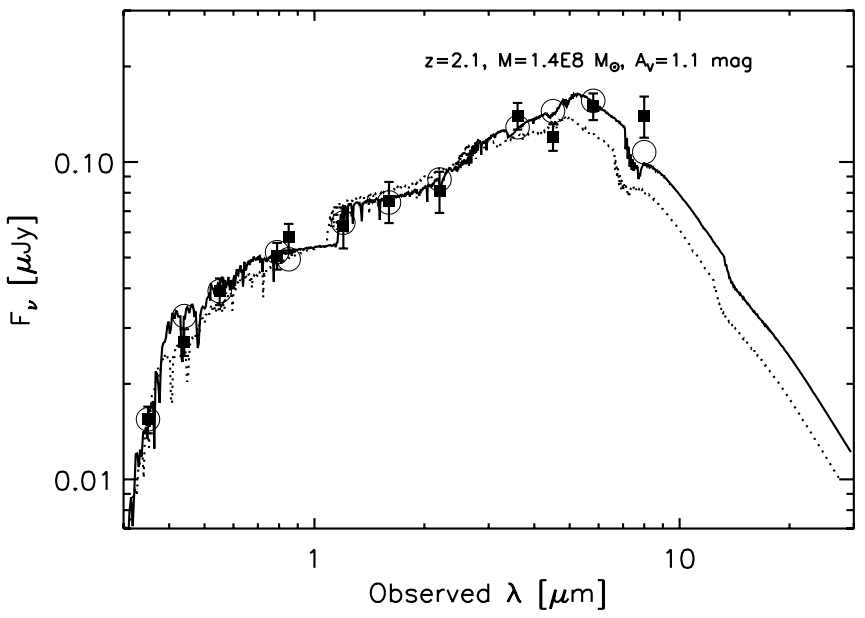

FIG. 2.- Stacked ultraviolet-to-IR flux SED of faint galaxies detected by ACS, but not detected individually by Spitzer IRAC in GOODS-S (solid line and data points). The SED is consistent with the sub- $L_{*, \mathrm{UV}}$ galaxy population at $z \sim 2.5$. The parameters of the stacked galaxy for GOODS-S are as shown in the legend. The convolution of the fit SED with the different passbands is shown with circles. The stacking in GOODS-N results in a best-fit redshift of $z \sim 1.9$, and the SED is shown as the dotted line (See text for details).

This implies $z>1.3$. Thus, even a qualitative comparison of the stacked flux densities with a typical galaxy SED results in a redshift range similar to what we estimate from the $\chi^{2}$ values. We adopt $1.9<z<2.7$ as the redshift range of these sources. The uncertainty in the derived stellar mass is about a factor of 2 , in the range $(1.3-3.0) \times 10^{8} M_{\odot}$.

For our best-fit template, the rest-frame $1500 \AA$ UV luminosity of the stacked galaxy is $1.8 \times 10^{9} L_{\odot}$, while its rest-frame $V$-band luminosity is $1.2 \times 10^{9} L_{\odot}$. Since $L_{*, \mathrm{UV}, z=3}=5.8 \times 10^{10}$ and $L_{*, \mathrm{~V}, z=3}=8 \times 10^{10} L_{\odot}$ (Steidel et al. 1999; Marchesini et al. 2007), it implies that these faint objects are galaxies which are about $\sim 30-60$ times fainter than the characteristic luminosity of field galaxies at these redshifts.

Although the exact redshift distribution of these faint galaxies is not known at this time, it is illustrative to estimate the fraction of the stellar mass density hidden in these objects. We assume that the $\sim 6000$ galaxies that we have stacked on are distributed in the range $1.9<z<2.7$ with a stellar mass of $1.4 \times 10^{8} M_{\odot}$. This corresponds to a comoving stellar mass density of $2 \times$ $10^{6} M_{\odot} \mathrm{Mpc}^{-3}$, which is a strong lower limit. Assuming that our stacked flux is typical for the $\sim 22,000$ ACS sources which are unmatched to IRAC sources, would imply a total stellar mass density in faint galaxies of $6.7 \times 10^{6} M_{\odot} \mathrm{Mpc}^{-3}$ at $z \sim 2.4$. For comparison, the stellar mass density in Lyman break galaxies at these redshifts is $3.6 \times 10^{7} \mathrm{M}_{\odot} \mathrm{Mpc}^{-3}$ (Dickinson et al. 2003b). Thus, the faint, sub- $L_{*}$ galaxy population could account for up to $20 \%$ of the total stellar mass density at $z \sim 2.4$.

\section{EXPECTED CLUSTERING}

We also measure the clustering of these sources to establish the amplitude of unresolved fluctuations at IRAC bands. Since we are interested in the anisotropy of IR light, to establish the power spectrum of fluctuations, we need the flux distribution within this population. The stacking analysis only allows us to establish either the total or the average flux of this sample. This forces us to make an estimate of the flux distribution and use that to predict clustering of unresolved fluctuations produced by these sources. The angular power spectrum of IR fluxes associated with these sources is determined using the same technique as described in Sullivan et al. (2007). We account for the flux distribution by 


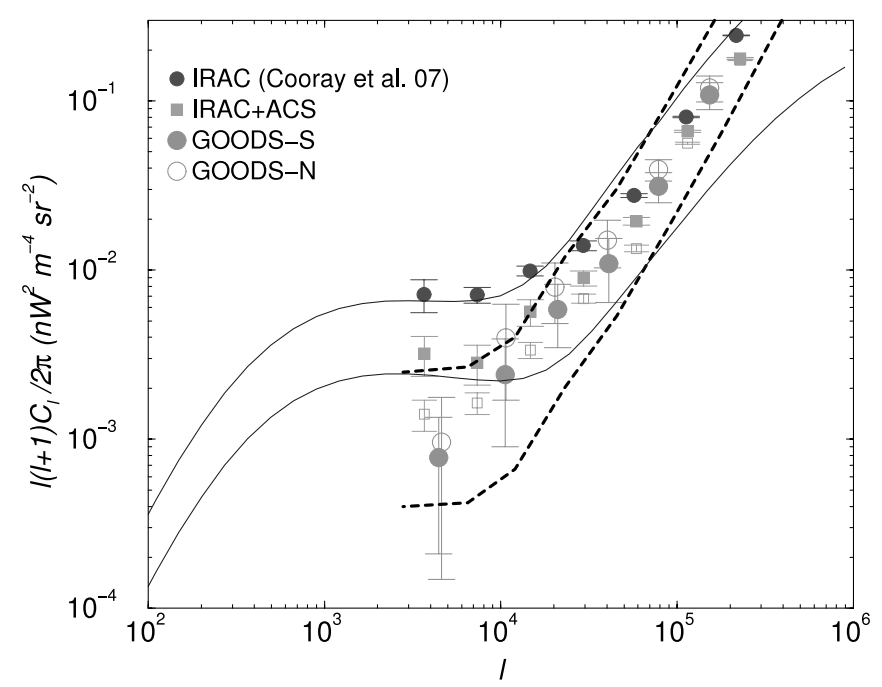

FIG. 3.-Top filled circles: Angular power spectrum of unresolved IR fluctuations in IRAC channel $1(3.6 \mu \mathrm{m})$ of GOODS-N field. The filled squares show the angular power spectrum that masks the locations of faint ACS sources that are undetected in IRAC. The same power spectrum published in Cooray et al. (2007) is shown with open squares, and the difference accounts for a new correction we have introduced here for the window function of the ACS source mask. With ACS sources masked, the fluctuation power spectrum amplitude is reduced at $\ell \sim 7 \times 10^{3}$ by a factor of about $2( \pm 0.6)$. The circles and dashed lines below show the predicted power spectrum for faint optical sources with their $3.6 \mu \mathrm{m}$ number counts distributed with two faint-end slopes: the open and filled circles are for clustering in GOODS-N and GOODS-S assuming the steeper slope for counts with $\alpha \sim 0.6$, while the dashed lines bracket the clustering for a flatter slope with $\alpha \sim 0\left(d N / d m \propto m^{\alpha}\right.$ when $\left.m>26\right)$. For reference, the two solid lines are predictions from clustering models of the faint source population with the range covering the uncertainty in model parameters (Sullivan et al. 2007). [See the electronic edition of the Journal for a color version of this figure.]

randomly assigning IR flux to the $\sim 6000$ optical source locations between 70 and $700 \mathrm{nJy}$ such that the total flux assigned is the same as the total flux measured for these sources from the stack. We assign fluxes such that the number counts trace the extrapolated slope from known IRAC counts down to $m_{\mathrm{AB}}(3.6 \mu \mathrm{m})$ of 24.7 mag. Given the uncertainty in the faint-end slope, we take the slope $\alpha\left(d N / d m \propto m^{\alpha}\right)$ to be either 0.6 or 0.0 . We tested how our predictions change with variations to these parameters and found consistent results within errors. Since the flux assignment is random, to get an average of the expected clustering, we randomize the assignments and the fluxes, and use a Monte Carlo approach to obtain the mean and variance of clustering.

We summarize our results in Figure 3, where we also compare with a direct measurement of the clustering of IR fluctuations and expectations based on the clustering models of Sullivan et al. (2007; solid lines). In Figure 3, we also show the IR fluctuation spectrum when the faint optical sources that we stack on are masked from the IRAC image with open squares (Cooray et al. 2007). The filled squares show the revised measurement after correcting for a bias associated with the window function introduced by the large mask. To understand this correction noted in Kashlinsky et al. (2007a), we note that the fluctuation spectrum measurable in Fourier (multipole) space in the presence of a mask is $\hat{C}_{\ell}=\sum_{\ell^{\prime}} W_{\ell \ell^{\prime}} C_{\ell^{\prime}}$, where $W_{\ell \ell^{\prime}}$ is the window function associated with the mask and $C_{\ell^{\prime}}$ is the power spectrum of interest. We recover the latter with measurements of $\hat{C}_{\ell}$ by first generating $W_{\ell \ell^{\prime}}$ associated with our mask (Appendix A of Hivon et al. 2002) and then iteratively inverting for $C_{\ell}$ using an inversion similar to Dodelson \& Gaztañaga (2000). The inversion agrees to $10 \%$ with a separate estimate of the angular power spectrum using a likelihood method where one maximizes the likelihood of an estimated $C_{\ell}^{\text {est }}$ with a model spectrum $C_{\ell}^{\text {inp }}$ using

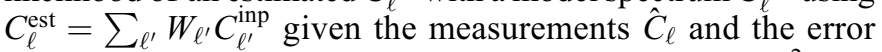
$\sigma_{\ell}$. This procedure can be described as minimizing the $\chi^{2}$ with $\chi^{2}=\left(\hat{C}_{\ell}-C_{\ell}^{\text {est }}\right)^{2} / \sigma_{\ell}^{2}$.

As shown in Figure 3, the difference between the fluctuation amplitude of unresolved IR fluctuations and the fluctuation amplitude with ACS sources masked is about a factor of $2( \pm 0.6)$ at $\ell \sim 7 \times 10^{3}$. Our measurements then suggest that, from this difference, up to $50 \%$ of IR fluctuations can be accounted by faint ACS sources. It could be that by accounting for further fainter optically sources, this fraction can be increased, but it is quite unlikely that $100 \%$ of IR fluctuations reported in Kashlinsky et al. $(2005,2007 b)$ are generated by $z>6.5$ galaxies hosting mostly Population III stars that are optically invisible.

The difference between IR fluctuations with and without ACS sources masked must be reproduced by the clustering of IR light produced by the same ACS sources. With flux assignments based on the stacking analysis, we find that the difference is reproduced (although these indirect estimates of the expected clustering IR fluctuations produced by faint ACS sources are uncertain due to uncertainties in the flux distribution). We conclude that irrespective of whether we measure the power spectrum in the IRB fluctuations after masking optical sources or whether we measure the power spectrum of faint optical galaxies with an average flux based on our stacking analysis, we find that the power in faint $\left(z_{\mathrm{AB}} \sim 27 \mathrm{mag}\right), z \sim 2.5$, galaxies accounts for at least $\sim 30 \%-$ $50 \%$ of the power in the IRB fluctuations on angular scales of $\sim 4^{\prime}$, where the measurement is not strongly sensitive to cosmic variance.

On similar angular scales, Kashlinsky et al. (2007a) find a power of $0.015 \mathrm{nW} \mathrm{m} \mathrm{mr}^{-1}$ for the optical galaxies with $z_{\mathrm{AB}}>26.6$ mag in Hubble Deep Field-North (HDFN)-E2, without a quantification of the uncertainty. They also demonstrate that the power increases as brighter galaxies are included. Forty percent of the galaxies that go into our stacking analysis are brighter than this threshold, and therefore the power they measure is a lower limit to our power spectrum shown in Figure 3. This can be compared to their measured value of $0.035 \mathrm{nW} \mathrm{m}^{-2} \mathrm{sr}^{-1}$ for the amplitude of power in the IRB fluctuations in the HDFN on the same angular scale/multipole moment. Making use of their estimate of the fluctuations associated with faint, optical galaxies at low redshifts, we find that Kashlinsky et al. (2007a, 2007 b) also find a $\sim 40 \%$ contribution from such sources to fluctuations at $\sim 4^{\prime}$ angular scales, although their contribution is ignored in their subsequent interpretation that assumes all fluctuations are from Population III stars before reionization. The analysis of the optical sources in GOODS-S is not presented in Kashlinsky et al. (2007a). The fundamental discrepancy between our two groups arises on larger angular scales, at $400^{\prime \prime}$ or larger, where they claim the power spectrum is rising. We are unable to constrain the power spectrum on such large angular scales $(\ell \sim 1500)$ due to the cosmic variance associated with the limited size of the GOODS fields used in our analysis, which becomes important at angular scales above $400^{\prime \prime}$.

\section{DISCUSSION}

The Kashlinsky et al. (2007c) interpretation for IR fluctuations involves $z>6.5$ galaxies with an IRB intensity of $\gtrsim 1 \mathrm{nW} \mathrm{m}^{-2}$ $\mathrm{sr}^{-1}$ at $3.6 \mu \mathrm{m}$. This is a large intensity given that all resolved sources, so far, lead to an IRB intensity between $\sim 6 \mathrm{nW} \mathrm{m}^{-2} \mathrm{sr}^{-1}$ (Sullivan et al. 2007; Fazio et al. 2004) and $\sim 10 \mathrm{nW} \mathrm{m}^{-2} \mathrm{sr}^{-1}$ (Levenson \& Wright 2008). To avoid individual detections of a large number of $z>6.5$ galaxies, such a high background 
intensity must then be hidden in a source population with a large surface density but with individual faint fluxes of around $10 \mathrm{nJy}$ for each source. Given the large surface density of the population, pixel-to-pixel intensity variations are a small fraction of the total intensity produced by those sources.

The alternative explanation is that a reasonable fraction of fluctuations arise from a population of sources that have a low surface density but are just below the Spitzer IRAC detection threshold. The results from the stacking analysis presented here suggest that faint optical galaxies, with an average source flux of around $130 \mathrm{nJy}$ at $3.6 \mu \mathrm{m}$, contribute between $\sim 30 \%$ and $50 \%$ of the power in the fluctuations on the largest angular scales that we are able to measure. The average IR absolute intensity produced by these sources is in the range $0.12-0.35 \mathrm{nW} \mathrm{m}^{-2} \mathrm{sr}^{-1}$ at $3.6 \mu \mathrm{m}$, in agreement with the Kashlinsky et al. (2005) estimate that the contribution from faint extragalactic sources is $\sim 0.15 \mathrm{nW} \mathrm{m}^{-2}$ $\mathrm{sr}^{-1}$. However, they claim that such faint objects cannot account for the strong clustering signal in unresolved IR light. We have demonstrated in this paper that a significant fraction of the fluctuations do arise from these faint galaxies. These sub- $L_{*}$ galaxies contribute up to $20 \%$ of the stellar mass density at $z \sim 2.5$ and can be described as a low-luminosity version of well-studied Lyman break galaxies.

While these sources do not fully explain all IR fluctuations measured with deep IRAC images, in Cooray et al. (2007) we placed a conservative upper limit that any contribution from $z>6.5$ sources must have an absolute intensity less than about $0.6 \mathrm{nW} \mathrm{m}^{-2} \mathrm{sr}^{-1}$. Beyond Spitzer IRAC, studies have also been conducted at lower near-IR wavelengths with the Near-Infrared Camera and Multi-Object Spectrometer (NICMOS). Although limited by the small field of view of NICMOS, they show that IR fluctuations at 1.6 and $1.25 \mu \mathrm{m}$ are more consistent with a $z<8$ origin than a high-redshift interpretation (Thompson et al. 2007a, 2007b). Beyond IR fluctuations, it will be interesting to further study the nature of the faint, dwarf galaxy population at $z=2-3$ to understand the contribution it makes toward bridging the systematic offset between the comoving stellar mass density and the integrated star formation rate density (Hopkins \& Beacom 2006).

We thank Mark Dickinson for helpful suggestions. We also acknowledge the contributions of various members of the GOODS team, who are associated with key aspects of data processing and handling. This research was funded by NASA grant NNX 07AG43G, NSF CAREER grant AST 06-45427, award number 1310310 from Spitzer for Archival Research, and program number HST-AR-11241.01-A by NASA through a grant from the Space Telescope Science Institute, which is operated by the Association of Universities for Research in Astronomy, Incorporated, under NASA contract NAS5-26555.
Aharonian, F., et al. 2006, Nature, 440, 1018

Babbedge, T. S. R., et al. 2006, MNRAS, 370, 1159

Bruzual, G., \& Charlot, S. 2003, MNRAS, 344, 1000

Capak, P., et al. 2004, AJ, 127, 180

Cooray, A., \& Sheth, R. 2002, Phys. Rep., 372, 1

Cooray, A., \& Yoshida, N. 2004, MNRAS, 351, L71

Cooray, A., et al. 2004, ApJ, 606, 611 (erratum 622, 1363 [2005]) 2007, ApJ, 659, L91

Dickinson, M., et al. 2003a, in The Mass of Galaxies at Low and High Redshifts,

ed. R. Bender \& A. Renzini (Berlin: Springer), 324 2003b, ApJ, 587, 25

Dodelson, S., \& Gaztañaga, E. 2000, MNRAS, 312, 774

Dwek, E., Arendt, R., \& Krennrich, F. 2005, ApJ, 635, 784

Giavalisco, M., et al. 2004, ApJ, 600, L93

Fazio, C. G., et al. 2004, ApJS, 154, 39

Fernandez, E., \& Komatsu, E. 2006, ApJ, 646, 703

Hauser, M. G., \& Dwek, E. 2001, ARA\&A, 39, 249

\section{REFERENCES}

Hopkins, A. M., \& Beacom, J. F. 2006, ApJ, 651, 142

Hivon, E., et al. 2002, ApJ, 567, 2

Kashlinsky, A., et al. 2004, ApJ, 608, 1

. 2005, Nature, 438, 45

2007a, ApJ, 666, L1

. 2007b, ApJ, 654, L5

. 2007c, ApJ, 654, L1

Levenson, L., \& Wright, E. L. 2008, ApJ, in press (arXiv: 0802.1239v1)

Madau, P., \& Silk, J. 2005, MNRAS, 359, L37

Marchesini, D., et al. 2007, ApJ, 656, 42

Matsumoto, T., et al. 2005, ApJ, 626, 31

Salvaterra, R., \& Ferrara, A. 2003, MNRAS, 339, 973

Santos, M. R., Bromm, V., \& Kamionkowski, M. 2002, MNRAS, 336, 1082

Steidel, C. C., et al. 1999, ApJ, 519, 1

Sullivan, I., et al. 2007, ApJ, 657, 37

Thompson, R., et al. 2007a, ApJ, 657, 669

. 2007b, ApJ, 666, 658 